\title{
Drop property on locally convex spaces
}

\author{
by \\ Ignacio Monterde and Vicente Montesinos (Valencia)
}

\begin{abstract}
A single technique provides short proofs of some results about drop properties on locally convex spaces. It is shown that the quasi drop property is equivalent to a drop property for countably closed sets. As a byproduct, we prove that the drop and quasi drop properties are separably determined.
\end{abstract}

1. Introduction. Let $B$ a non-empty closed bounded convex subset of a Banach space $X$. By the $d r o p$ induced by a point $a \in X \backslash B$ we mean the set $D(a, B):=\operatorname{conv}(\{a\} \cup B)$. Daneš showed in [Da72] that if $A$ is a non-empty closed subset of $X$ at positive distance of the closed unit ball $B:=B_{X}$, then there exists $a \in A$ such that $D\left(a, B_{X}\right) \cap A=\{a\}$. In [Da85] he showed that the same is true if $B$ is any non-empty closed convex and bounded subset of $X$. This is referred to as the Daneš drop theorem. A closed convex and bounded subset $B$ of $X$ is said to have the drop property if for any non-empty closed subset $A$, there exists a point $a \in A$ such that $D(a, B) \cap A=\{a\}$. Rolewicz proved in [Ro85] that if $X$ is reflexive then $B_{X}$ has the drop property. The second named author proved in [Mo87] that $a$ Banach space is reflexive if and only if it can be renormed to have the drop property. In [Ku87] it was proved that every weakly compact convex subset of a Banach space has the drop property.

Different versions of the drop property in locally convex spaces were considered by several authors. A closed convex bounded subset $B$ of a locally convex space $(E, \mathcal{T})$ is said to have the weak drop (resp. the quasiweak drop) property if for every non-empty weakly sequentially closed (resp. weakly closed) subset $A$ of $E$ disjoint from $B$ there exists $a \in A$ such that $D(a, B) \cap A=\{a\}$. Obviously, the weak drop property implies the quasi-weak drop property. Moreover, Qiu proved that the converse does not hold (see

2000 Mathematics Subject Classification: Primary 46B20; Secondary 46B50.

Key words and phrases: drop property, quasi-drop property, weak compactness, reflexivity.

Supported in part by Project MTM2005-08210 and the Universidad Politécnica de Valencia. 
[Q03, Ex. 3.1]). The same author gave sufficient conditions for both drop properties by proving that weak sequential compactness implies the weak drop property [Q03, Th. 2.1] and that weak countable compactness implies the quasi-weak drop property [Q04, Th. 2.1]. In this paper we present short proofs of those results (for arbitrary locally convex topologies further than the weak topology) by underlying their dependence from the classical Daneš drop theorem.

Definition 1. A closed convex bounded subset $B$ of a locally convex space $(E, \mathcal{T})$ is said to have the $d r o p$ (resp. quasi drop) property if it is nonempty and for every non-empty sequentially closed (resp. closed) subset $A$ of $E$ disjoint from $B$ there exists $a \in A$ such that $D(a, B) \cap A=\{a\}$.

A subset $A$ of a locally convex space $(E, \mathcal{T})$ is countably closed (resp. sequentially closed) if it contains the closure of each of its countable subsets (resp., the limit of every sequence in $A$ which converges in $E$ ). The following implications are clear:

$$
\text { closed } \Rightarrow \text { countably closed } \Rightarrow \text { sequentially closed. }
$$

However, none of the converse implications hold true. For instance, take $\left(\ell_{\infty}[0,1], \mathcal{T}_{p}\right)$, the space of bounded functions in $[0,1]$ endowed with the topology of pointwise convergence. Consider the set $A:=\left\{f \in \ell_{\infty}:\|f\|_{\infty} \leq 1\right\}$ and let $B$ be the subset of $A$ consisting of all countably supported elements. Then $B$ is countably closed, but not closed, since its closure is $A$. On the other hand, the subset $\ell_{1}$ of $\left(\ell_{\infty}^{*}, \sigma\left(\ell_{\infty}^{*}, \ell_{\infty}\right)\right)$ is sequentially closed (by the Schur lemma) but not countably closed, since the countable set $\left\{e_{n} ; n \in \mathbb{N}\right\}$ $\cup\{0\}$ is not weakly compact in $\ell_{1}$. A bounded set with the same property is the closed unit ball of $\ell_{1}$ as a subset of $\left(\ell_{\infty}^{*}, \sigma\left(\ell_{\infty}, \ell_{\infty}\right)\right)$.

Qiu studied and separated the concepts of drop property dealing (in the weak topology) with sequentially closed subsets (weak drop property) and with closed subsets (weak quasi drop property). It is natural to ask about a seemingly intermediate concept, the drop property dealing with countably closed subsets. We show here that this apparently new property is indeed the same as the quasi drop property.

2. Results. The key point in our proofs is the possibility to embed a subset of a locally convex space in a Banach space. An absolutely convex and bounded subset $U$ of a locally convex space $(E, \mathcal{T})$ is called a Banach disc if $E_{U}:=\operatorname{span}(U)$, endowed with the norm $\|\cdot\|_{U}$ defined by the Minkowski gauge of $U$, is a Banach space. Observe that $\left(E_{U},\|\cdot\|_{U}\right)$ continuously embeds in $(E, \mathcal{T})$. In $[\mathrm{Fl} 80]$ the following result is proved:

TheOREM 2 ([Fl80, p. 17]). Every convex relatively countably compact subset $A$ of a locally convex space $E$ is contained in a Banach disc $U \subset E$. 
A more general version of this result for the class of convex sets which are convex-compact (a concept due to Šmulian, see [Ko69, §24]), has been proved by the present authors in $[\mathrm{MM}]$. Using Theorem 2 we can easily reduce [Q03, Th. 2.1], not only for the weak topology but, in general, for every locally convex topology, to the classical Daneš theorem.

THEOREM 3. Every non-empty closed convex and sequentially compact subset $B$ of a locally convex space $(E, \mathcal{T})$ has the drop property.

Proof. Let $A$ be a sequentially closed subset of $E$ disjoint from $B$. Let $a_{0} \in A$. It is easy to see that $D\left(a_{0}, B\right)$ is closed and sequentially compact, so it is contained in a Banach disc $U$. Let $d_{U}$ be the metric induced in $E_{U}$ by $\|\cdot\|_{U}$. Suppose that $d_{U}\left(A \cap D\left(a_{0}, B\right), B\right)=0$. Then we can find two sequences $\left(a_{n}\right)$ in $A \cap D\left(a_{0}, B\right)$ and $\left(b_{n}\right)$ in $B$ such that $\left\|a_{n}-b_{n}\right\|_{U} \rightarrow 0$ (so $a_{n}-b_{n} \stackrel{\mathcal{T}}{\rightarrow} 0$ ). Since $B$ is $\mathcal{T}$-sequentially compact, we can extract a subsequence $\left(b_{n_{i}}\right)$ such that $b_{n_{i}} \stackrel{\mathcal{T}}{\rightarrow} b \in B$. But then $a_{n_{i}} \stackrel{\mathcal{T}}{\rightarrow} b$, so $b \in A$, since $A$ is $\mathcal{T}$-sequentially closed, a contradiction. Therefore $d_{U}\left(A \cap D\left(a_{0}, B\right), B\right)>0$. Obviously, $A \cap D\left(a_{0}, B\right)$ is $\|\cdot\|_{U}$-closed. Applying the Daneš theorem in $\left(E_{U},\|\cdot\|_{U}\right)$, we know that there exists $a_{1} \in A \cap D\left(a_{0}, B\right)$ such that

$$
D\left(a_{1}, B\right) \cap A \cap D\left(a_{0}, B\right)=\left\{a_{1}\right\} .
$$

Since $a_{1} \in D\left(a_{0}, B\right)$, we can easily verify that $D\left(a_{1}, B\right) \subset D\left(a_{0}, B\right)$, so

$$
D\left(a_{1}, B\right) \cap A=\left\{a_{1}\right\} .
$$

[Q04, Theorem 2.1], again for every locally convex topology, can also be proved in a very simple way by using arguments similar to the ones in the former proof.

THEOREM 4. Every non-empty closed convex and countably compact subset $B$ of a locally convex space $(E, \mathcal{T})$ has the quasi drop property.

Proof. Let $A$ be a closed subset of $E$ disjoint from $B$ and let $a_{0} \in A$. Since $D\left(a_{0}, B\right)$ is closed and countably compact, it is contained in a Banach disc $U$. As above, suppose that $d_{U}\left(A \cap D\left(a_{0}, B\right), B\right)=0$ and take two sequences $\left(a_{n}\right)$ in $A \cap D\left(a_{0}, B\right)$ and $\left(b_{n}\right)$ in $B$ such that $\left\|a_{n}-b_{n}\right\|_{U} \rightarrow 0$ (so $\left.a_{n}-b_{n} \stackrel{\mathcal{T}}{\rightarrow} 0\right)$. Since $B$ is $\mathcal{T}$-countably compact, we can extract a subnet $\left(b_{n_{i}}\right)$ such that $b_{n_{i}} \stackrel{\mathcal{T}}{\rightarrow} b \in B$. But then $a_{n_{i}} \stackrel{\mathcal{T}}{\rightarrow} b$, so $b \in A$, since $A$ is $\mathcal{T}$-closed, a contradiction. Again the Daneš theorem in $\left(E_{U},\|\cdot\|_{U}\right)$ implies our result.

Observe that, from the above proof, the result holds even if $A$ is supposed to be just countably closed (indeed, the range of the net $\left(a_{n_{i}}\right)$ is countable). Apparently, non-empty closed convex and countably compact subsets of a locally convex space enjoy a stronger property than the quasi drop property, namely the possibility to find "drop points" for every non-empty countably closed subset of $(E, \mathcal{T})$ disjoint from $B$ (we showed above that there are 
countably closed subsets of a locally convex space which are not closed). We shall prove below that these two seemingly different drop properties coincide. To begin with, we need a simple lemma.

Lemma 5. Let $(X,\|\cdot\|)$ be a normed space and $B$ a non-empty closed convex and bounded subset of $X$. Let $a \in X$ be such that $a \notin B$. Then, for every $x \in D(a, B)$ with $x \neq a$, we have $\operatorname{dist}(x, B)<\operatorname{dist}(a, B)$, where dist denotes the distance in $X$ induced by $\|\cdot\|$.

Proof. Let $d:=\operatorname{dist}(a, B)(>0)$. There exists a sequence $\left(b_{n}\right)$ in $B$ such that $\left\|a-b_{n}\right\|<d+1 / n$ for all $n \in \mathbb{N}$. Take $x \in D(a, B), x \neq a$. Then there exist $0 \leq \lambda<1$ and $b \in B$ such that $x=\lambda a+(1-\lambda) b$. Observe that $y_{n}=\lambda b_{n}+(1-\lambda) b \in B$, since $B$ is convex. If $\lambda=0$ then $x=b \in B$, so $\operatorname{dist}(x, B)=0<d=\operatorname{dist}(a, B)$ and we are done. Otherwise, for $n \in \mathbb{N}$,

$$
\begin{aligned}
\operatorname{dist}(x, B) \leq\left\|x-y_{n}\right\| & =\left\|\lambda a+(1-\lambda) b-\lambda b_{n}-(1-\lambda) b\right\| \\
& =\lambda\left\|a-b_{n}\right\|<\lambda(d+1 / n) .
\end{aligned}
$$

Since this is true for every $n$, we get $\operatorname{dist}(x, B) \leq \lambda d<d=\operatorname{dist}(a, B)$.

Theorem 6. Let $(E, \mathcal{T})$ be a locally convex space and $B$ a closed, convex and bounded subset of $E$ with the quasi drop property. Then, for every nonempty countably closed set $A$ disjoint from $B$, there exists $a \in A$ such that $D(a, B) \cap A=\{a\}$.

Proof. We shall argue by contradiction. Let $B$ be a closed, convex and bounded subset of $E$ with the quasi drop property and suppose that we can find $A$, a non-empty countably closed subset of $X$ disjoint from $B$, such that there is no $x \in A$ satisfying $D(x, B) \cap A=\{x\}$. Fix $a \in A$ and let $D:=\overline{\Gamma(D(a, B))}$ be the absolutely convex and closed hull of $D(a, B)$, a disk in $E$. Let dist be the $\|\cdot\|_{D}$-distance in the normed space $\left(E_{D},\|\cdot\|_{D}\right)$.

We shall construct a sequence $\left(x_{n}\right)$ in $A$ with the following properties.

(i) $x_{1}:=a$.

(ii) $\left(x_{n}\right)$ is a stream, i.e., $x_{n+1} \in D\left(x_{n}, B\right)$ for all $n \in \mathbb{N}$, and $x_{n+1} \neq x_{n}$ for all $n \in \mathbb{N}$.

(iii) $\operatorname{dist}\left(x_{n+1}, B\right)<\operatorname{dist}\left(D\left(x_{n}, B\right) \cap A, B\right)+1 / n$ for all $n \in \mathbb{N}$.

This will be done by induction. Let us start by taking $x_{1}:=a$. Assume now that, for some $i \in \mathbb{N}$, elements $x_{1}, \ldots, x_{i}$ in $A$ have already been defined such that (i)-(iii) above hold for $n=1, \ldots, i-1$. Compute then $\operatorname{dist}\left(D\left(x_{i}, B\right) \cap\right.$ $A, B)$ and choose $x_{i+1} \in D\left(x_{i}, B\right) \cap A$ such that

$$
\left(\operatorname{dist}\left(D\left(x_{i}, B\right) \cap A, B\right) \leq\right) \operatorname{dist}\left(x_{i+1}, B\right)<\operatorname{dist}\left(D\left(x_{i}, B\right) \cap A, B\right)+1 / i .
$$

The fact that $D\left(x_{i}, B\right) \cap A \neq\left\{x_{i}\right\}$ allows us to choose $x_{i+1} \neq x_{i}$. This finishes the construction. 
The set $B$ has the quasi drop property, hence, if $\left\{x_{i} ; i \in \mathbb{N}\right\}$ is $\mathcal{T}$-closed, there is some $x_{n}$ such that $D\left(x_{n}, B\right) \cap\left\{x_{i} ; i \in \mathbb{N}\right\}=\left\{x_{n}\right\}$, a contradiction. Then, $\left\{x_{n} ; n \in \mathbb{N}\right\}$ is not $\mathcal{T}$-closed. Let $\widetilde{x} \in \overline{\left\{x_{n} ; n \in \mathbb{N}\right\}} \backslash\left\{\left\{x_{n} ; n \in \mathbb{N}\right\}\right.$. Since $A$ is countably closed, we have $\widetilde{x} \in A$. Moreover, $\widetilde{x} \in{\overline{\left\{x_{m} ; m \geq n\right\}}}^{\mathcal{T}}$ for all $n \in \mathbb{N}$. Obviously, the set $D\left(x_{n}, B\right)$ is $\mathcal{T}$-closed, so $\widetilde{x} \in{\overline{D\left(x_{n}, B\right)}}^{\mathcal{T}} \cap A=$ $D\left(x_{n}, B\right) \cap A$ for all $n \in \mathbb{N}$. In particular, $D(\widetilde{x}, B) \subset D\left(x_{n}, B\right)$ for all $n \in \mathbb{N}$. Let $x \in D(\widetilde{x}, B) \cap A$. Then, since $x \in D\left(x_{n+1}, B\right) \subset D\left(x_{n}, B\right)$, we have, using Lemma 5 ,

$$
\begin{aligned}
\operatorname{dist}\left(D\left(x_{n}, B\right) \cap A, B\right) & \leq \operatorname{dist}(x, B) \leq \operatorname{dist}\left(x_{n+1}, B\right) \\
& <\operatorname{dist}\left(D\left(x_{n}, B\right) \cap A, B\right)+1 / n .
\end{aligned}
$$

We have, too,

$$
\begin{aligned}
\operatorname{dist}\left(D\left(x_{n}, B\right) \cap A, B\right) & \leq \operatorname{dist}(\widetilde{x}, B) \leq \operatorname{dist}\left(x_{n+1}, B\right) \\
& <\operatorname{dist}\left(D\left(x_{n}, B\right) \cap A, B\right)+1 / n .
\end{aligned}
$$

Then

$$
0 \leq \operatorname{dist}(\widetilde{x}, B)-\operatorname{dist}(x, B)<1 / n \quad \text { for all } n \in \mathbb{N},
$$

hence, for all $x \in D(\widetilde{x}, B) \cap A$ we have $\operatorname{dist}(x, B)=\operatorname{dist}(\widetilde{x}, B) \neq 0$. In view of Lemma 5 , it follows that $x=\widetilde{x}$ for every $x \in D(\widetilde{x}, B) \cap A$, i.e., $D(\widetilde{x}, B) \cap A=\{\widetilde{x}\}$, and we arrive at a contradiction.

A byproduct of the former theorem is that drop and quasi drop properties are separably determined, which is not evident from the very definition, since no metrizability nor, more generally, angelicity are present in this context. We have the following two results.

Corollary 7. Let $(E, \mathcal{T})$ be a locally convex space. Let $B$ be a closed convex and bounded subset of $E$. Then $B$ has the quasi drop property if and only if $B \cap S$ has the quasi drop property for every closed separable subspace $S \subset E$.

Proof. One direction is quite obvious. Indeed, suppose that $B$ has the quasi drop property and let $S$ be a closed separable subspace of $E$. Let $A$ be a closed subset of $S$ disjoint from $B$. Then $A$ is closed in $E$ and we can find $a \in A$ such that $D(a, B) \cap A=\{a\}$. Therefore

$$
D(a, B \cap S) \cap A=\{a\} .
$$

Assume now that $B \cap S$ has the quasi drop property for every closed separable subspace $S \subset E$ and assume that $B$ does not have the quasi drop property in $E$. Let $A$ be a closed subset of $E$ such that no $x \in A$ has the property that

$$
D(x, B) \cap A=\{x\} .
$$


As in the proof of Theorem 6, we can find a sequence $\left(x_{n}\right)$ in $A$ with the properties listed there. Let $S:=\overline{\operatorname{span}\left(x_{n}\right)}$, a closed separable subspace of $E$. The set $B \cap S$ has the quasi drop property. Then $\left\{x_{n} ; n \in \mathbb{N}\right\}$ is closed neither in $S$, nor in $E$. Find $\widetilde{x} \in A$ as in the proof of Theorem 6 and continue that proof to get $D(\widetilde{x}, B) \cap A=\{\widetilde{x}\}$, a contradiction.

An analogous proof works for the drop property.

Corollary 8. Let $(E, \mathcal{T})$ be a locally convex space. Let $B$ be a closed convex and bounded subset of $E$. Then $B$ has the drop property if and only if $B \cap S$ has the drop property for every closed separable subspace $S \subset E$.

Proof. Again, one direction is almost obvious. For the other implication, assume that $B \cap S$ has the drop property for every separable subspace $S$ of $E$. If $B$ fails to have the drop property, we can find a sequentially closed set $A \subset E$ such that no $x \in A$ satisfies $D(x, B) \cap A=\{x\}$. As in the previous proof we get a sequence $\left(x_{n}\right)$ in $A$ with the properties listed in Theorem 6. Let $S:=\overline{\operatorname{span}\left(x_{n}\right)}$. The set $\left\{x_{n} ; n \in \mathbb{N}\right\}$ is sequentially closed neither in $S$, nor in $E$. Then there exists a subsequence $\left(x_{i}\right)$ of $\left(x_{n}\right)$ which converges to a point $\widetilde{x}$ in $A$ (actually $x_{n} \rightarrow \widetilde{x}$ ). Now it is easy to prove that $D(\widetilde{x}, B) \cap A=\{\widetilde{x}\}$, a contradiction.

Acknowledgements. We thank an anonymous referee for his/her suggestions that helped to improve the final version of the manuscript.

\section{References}

[Da72] J. Daneš, A geometric theorem useful in nonlinear functional analysis, Boll. Un. Mat. Ital. 6 (1972), 369-372.

[Da85] - Equivalence of some geometric and related results of nonlinear functional analysis, Comment. Math. Univ. Carolin. 26 (1985), 443-454.

[F180] K. Floret, Weakly Compact Sets, Lecture Notes in Math. 801, Springer, 1980.

[Ko69] G. Köthe, Topological Vector Spaces I, Springer, 1969.

[Ku87] D. N. Kutzarova, On the drop property of convex sets in Banach spaces, in: Constructive Theory of Functions'87, Sofia, 1988, 283-287.

[MM] I. Monterde and V. Montesinos, Convex-compact sets and Banach discs, to appear.

[Mo87] V. Montesinos, Drop property equals reflexivity, Studia Math. 87 (1987), 93-100.

[Q02] J. H. Qiu, Local completeness and drop theorem, J. Math. Anal. Appl. 266 (2002), 288-297.

[Q03] -, On weak drop property and quasi-weak drop property, Studia Math. 156 (2003), 189-202.

[Q04] -, Weak countable compactness implies quasi-weak drop property, ibid. 162 (2004), 175-182. 
[Ro85] S. Rolewicz, On drop property, ibid. 85 (1987), 27-35.

Ignacio Monterde

Universidad Politécnica de Valencia

E-mail: nachomonterde@gmail.com
Vicente Montesinos Instituto de Matemática Pura y Aplicada Universidad Politécnica de Valencia Camino de Vera, s/n 46022 Valencia, Spain

E-mail: vmontesinos@mat.upv.es

Received June 6, 2006

Revised version December 28, 2007 